\title{
Spatial competition with concave transport costs
}

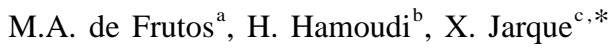 \\ ${ }^{a}$ Universidad Carlos III de Madrid, Madrid, Spain \\ ${ }^{\mathrm{b}}$ Universidad Europea de Madrid, Madrid, Spain

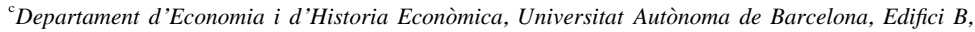 \\ 08193 Bellaterra, Spain
}

Received 22 December 1999; accepted 27 February 2001

\begin{abstract}
We study the location-then-price game played by two firms in a circular market when consumers face non-linear transport costs. We show that for any convex transport cost function there exists a concave one such that the location-then-price games induced by these functions are strategically equivalent. Further, we provide a sufficient condition to guarantee that a similar equivalence result holds under oligopolistic competition among equidistant firms. (c) 2002 Elsevier Science B.V. All rights reserved.
\end{abstract}

Keywords: Spatial competition; Equilibria; Oligopoly

JEL classification: C72; D43; L13

\section{Introduction}

Salop's (1979) circular market model has been widely used to study localized competition (see, for instance, Anderson, 1986 and Economides, 1989). Competition on the circular market can be interpreted as competition around a lake or as competition by companies offering daily services at a particular time of the day. As with the linear market model, contributions using the circular model have

*Corresponding author Tel : +34-935-811-802; fax: +34-935-812-012

E-mail address: xavier jarque@uab es (X Jarque) 
generally considered specific convex transport cost functions whenever a multistage game is proposed.

The purpose of this paper is to show that the convexity or concavity of the transport cost function is not relevant for the competitive decisions of the firms when the market is circular. More precisely, we show that for a given increasing convex transport cost function there exists an increasing concave one such that the location-then-price games induced by these functions are strategically equivalent.

In a circular market with two firms competing to sell their products, a consumer located at $x, x \in[0,1)$, purchasing a product from firm located at $x_{i}$ at price $p_{i}$ obtains utility

$$
u\left(x, x_{i}\right)=-p_{i}-F\left(z_{i}\right),
$$

where $z_{i}$ denotes the distance between $x$ and $x_{i}$ and where $F$ stands for the transport cost of travelling $z_{i}$, with $F(0)=0$. Clearly, if both firms always charge the same prices then a transformation in the transport cost function from convex to concave results in a monotone transformation in the consumers utilities. Thus, consumer theory tells us that the consumers decisions will not be modified by this transformation, and, consequently, the firm's competitive decisions will be not modified either. However, whenever firms charge different prices then a transformation in the transport cost does not induce a monotone transformation in the consumers' utilities. Nevertheless, the symmetry of the circular market allows one to find for any consumer $x$ facing $C$, another consumer $\bar{x}$ facing $T$ whose utility is a monotone transformation of the utility of $x$. Consumer $\bar{x}$ facing $T$ will hence buy from the same firm that consumer $x$ does, facing $C$. The above argument cannot be extended to the linear market model.

The main result in the paper is hence an equivalence (or duality) result showing that the game induced by a given convex transport cost $C$ is strategically equivalent to the game induced by a certain concave transport cost $T$. This result may have some interest inasmuch as until now it has not been known how to incorporate concave transport costs into the analysis of spatial competition. Furthermore, the result has several implications. On one hand, it ensures that, for the circular market, neither the existence of equilibrium nor the pattern of product differentiation rely on the convexity of transport costs. On the other hand, it allows one to translate any existence or uniqueness result for particular convex cost functions into the corresponding result for the appropriate concave cost functions.

In a previous work (see De Frutos et al., 1999) we show that only two cost functions from the linear quadratic family $-C(z)=z^{2}$ and $T(z)=z-z^{2}-$ ensure existence of a perfect equilibrium in pure strategies. One might think that this result deprives our current results of generality. We do not take this view. Rather we think that the contribution of this paper is to show that relaxing the widely used assumption of convexity in the transport cost has no impact on the results. In 
particular, this implies that when searching for transport cost functions that yield existence of equilibrium one can just focus on the family of convex functions.

The equivalence result hinges on the assumption of duopolistic competition. Nevertheless, some of the insights from this analysis can be extended to oligopolistic competition. In particular, under the proviso of limited consumer information (consumers make comparisons only between the two firms that are closest to them and then purchase from one of them), we provide an equivalence result for Salop's original model.

The paper is organized as follows. Section 2 presents the model. The equivalence result is proved in Section 3. Finally, Section 4 analyzes oligopolistic competition.

\section{The model}

In a circular market of length 1 there are two sellers, located on the circumference of the circle at $x_{1}$ and $x_{2}$, who charge mill prices $p_{1}$ and $p_{2}$, respectively. Both sellers supply a homogeneous product at zero marginal cost.

A continuum of consumers are spread uniformly with unit density on the circle. Each consumer purchases one unit of good and has a reservation price large enough so that she will always prefer to buy the good. Consumers will buy from the seller with the lowest delivered or full price, mill price plus transport cost.

Transport costs are assumed to be non-negative, continuous and increasing in the (relative) distance, denoted by $z$, with zero cost when travelling zero distance. ${ }^{1}$

We study a two-stage game where firms choose first locations and then compete in prices. We assume, w.l.o.g., that firm 1 locates at $x_{1}=0$ and that firm 2 chooses a location in the interval $[0,1 / 2]$. For a given strategy profile (a pair of prices and locations), the demand for the product of firm $i, D_{i}$, will be the mass of consumers that prefer to buy from firm $i$.

A consumer who faces the same full price from the two firms will be called an indifferent consumer. Since the indifferent consumers determine the market boundaries between the firms, we first study their locations. Under convex transport cost we have the following:

If there exists only one indifferent consumer then either she is located at $1 / 2$ and then $D_{1}=1$, or she is located at $1 / 2+x_{2}$ and then $D_{1}=0$.

If there are two indifferent consumers, denoted by $r$ and $s$, then $r \in(1 / 2$, $\left.1 / 2+x_{2}\right)$ and $s \in\left((0,1 / 2) \cup\left(1 / 2+x_{2}, 1\right)\right)$.

More precisely, the dynamic of the market boundaries as firm 1 increases its price is the following: one indifferent consumer moves monotonically from $1 / 2$

\footnotetext{
${ }^{1}$ This spatial setting is completely analogous to a model of product differentiation where $x_{1}$ and $x_{2}$ are two different brands or product types and where the transport cost is now interpreted as the disutility of not getting the most preferred product type
} 


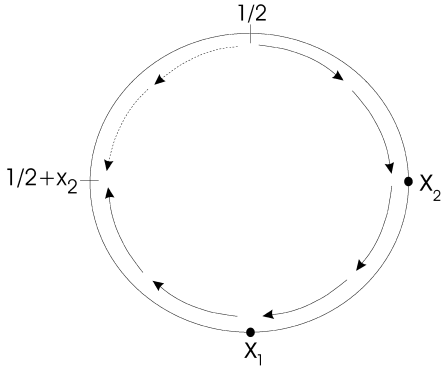

(a)

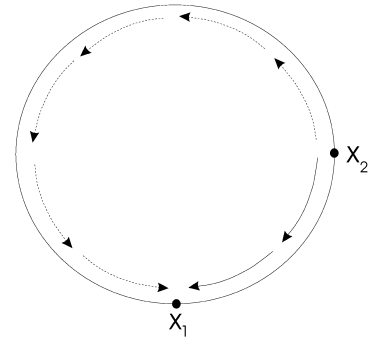

(b)

Fig 1 Dynamics of the indifferent consumers when (a) transport costs are convex and (b) transport costs are concave

towards $1 / 2+x_{2}$ clockwise, whereas the other indifferent consumer moves monotonically from $1 / 2$ towards $1 / 2+x_{2}$ counter clockwise. Thus, under convex transport costs and positive demands, the market of firm $i$ is an arc of the circle such that it always contains the point $1 / 2+x_{j}$, and it never contains the point $1 / 2+x_{i}$. Fig. 1(a) illustrates this dynamic.

The location of the indifferent consumers under concave transport cost is as follows:

If there exists only one indifferent consumer then either she is located at $x_{2}$ and then $D_{1}=1$, or she is located at 0 and then $D_{1}=0$.

If there are two indifferent consumers, denoted by $r$ and $s$, then $r \in\left(0, x_{2}\right)$ and $s=\left(x_{2}, 1\right)$.

The dynamic of the market boundaries as firm 1 increases its price is the following: one indifferent consumer moves monotonically from $x_{2}$ towards 0 clockwise, whereas the other indifferent consumer moves monotonically from $x_{2}$ towards 1 counter clockwise. Therefore, in the concave case, the market of firm $i$ is an arc of the circle such that it always contains the point $x_{i}$, and it never contains the point $x_{j}$. Fig. 1(b) illustrates this dynamic.

In Section 5, we will consider a variant of this model which is inspired by Salop's oligopolistic model (1979). The details of this variant will be set out in Section 5 .

\section{An equivalence result}

In a circular market of length 1 , the maximum distance that a consumer may travel is $1 / 2$ and the minimum distance is zero. Thus, for an arbitrary cost function $F$, all pairs $(z, F(z))$ lie in a rectangle with length $1 / 2$ and height $F(1 / 2)$. Consider 
this rectangle but with the origin located at the point $(1 / 2, F(1 / 2))$. With this new origin, the graph of $F(z)$ coincides with the graph that the function $\tilde{F}(z)=F(1$ / $2)-F(1 / 2-z)$ generates when the origin is located at $(0,0)$. Using this geometrical argument, it is easy to see that for any convex transport cost $C$ there exists a unique function $T$ such that $T$ coincides with $C$ if the above change in the origin is made. Formally, $T(z)=C(1 / 2)-C(1 / 2-z)$. Note that $T$ is concave with $T(0)=C(0)=0$ and $T(1 / 2)=C(1 / 2)$. Fig. 2 illustrates these statements.

We will use this result to show that for any arbitrary convex transport cost there exists a (unique) concave transport cost such that the two location-then-price games induced by these two functions are 'equivalent.'

To make the notion of 'equivalent games' precise let us focus on the second stage of the game or price subgame. Let $G\left(F ; x_{1}, x_{2}\right)$ represent the price subgame induced by the cost function $F$ and by the pair of locations $\left(x_{1}, x_{2}\right)$. Let $D_{i}^{G}(p)$ denote the market for firm $i$ when $p=\left(p_{1}, p_{2}\right)$ is played in the game $G$.

Definition 1. Two price subgames $G\left(F ; x_{1}, x_{2}\right)$ and $G^{\prime}\left(F^{\prime} ; x_{1}^{\prime}, x_{2}^{\prime}\right)$ are fully equivalent iff for every firm $i$, and for any strategy profile $p \in \Re_{+}^{2}$, it is the case that

$$
D_{i}^{G}(p)=D_{i}^{G^{\prime}}(p)
$$

Finally, two location-then-price games are fully equivalent if all their price subgames are fully equivalent. This notion of equivalence is taken from Myerson (1991) where it is defined in a more general framework. ${ }^{2}$

To illustrate the notion of equivalence consider the following example. Let the firms' locations be $(0,1 / 4)$. Consider the price subgames $G\left(10 z^{2} ; 0,1 / 4\right)$ and

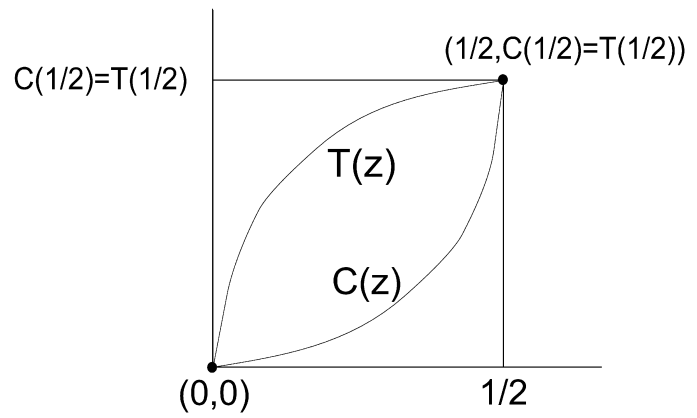

Fig 2 Transport costs

\footnotetext{
${ }^{2}$ Two games in strategic form $\Gamma\left(N,\left(S_{i}\right)_{i \in N,}\left(u_{i}\right)_{i \in N}\right)$ and $\Gamma^{\prime}\left(N,\left(S_{i}\right)_{i \in N}\left(u_{i}^{\prime}\right)_{i \in N}\right)$ are fully equivalent iff, for every player $i$, there exist numbers $A_{i}$ and $B_{i}$ such that for any strategy profile $s=\left(s_{i}\right)_{i \in N}$ it is the case that $u_{i}^{\prime}(s)=A_{i} u_{i}(s)+B_{i}$
} 
$G^{\prime}\left(10\left(z-z^{2}\right)\right.$; $\left.0,1 / 4\right)$. For the strategy profile $\bar{p}=(2,3)$, the indifferent consumers are located at 0.32 and 0.56 if firms play $G$, and at 0.19 and 0.42 if firms play $G^{\prime}$. We then have $D_{1}^{G}(\bar{p})=D_{1}^{G^{\prime}}(\bar{p})=0.77$ and, consequently, $D_{2}^{G}(\bar{p})=$ $D_{2}^{G^{\prime}}(\bar{p})=0.23$. It is important to emphasize that while market demands coincide in the two games (i.e., the mass of consumers buying from each firm is the same for both transport costs), market segments do not (i.e., the locations of the consumers buying from each firm differ).

In order to show that the result in this example holds true for any pair of locations and prices we first provide an auxiliary result.

Lemma 1. The games $G\left(F ; x_{1}, x_{2}\right)$ and $G^{\prime}\left(F ; x_{1}^{\prime}, x_{2}^{\prime}\right)$ are fully equivalent if and only if the distance between the two firms is the same in both games, where $F$ is either convex or concave.

Proof. We first suppose that $F$ is a convex function. By the symmetry of the market, let us assume, w.l.o.g., that $x_{1}=x_{1}^{\prime}=0$, and $0 \leq x_{2} \leq x_{2}^{\prime} \leq 1 / 2$. Sufficiency follows straightforwardly because same distance implies $x_{2}^{\prime}=x_{2}$. We prove necessity by way of contradiction. Consider a pair of strategies such that $y=1 / 2$ is an indifferent consumer when firms are located at $\left(0, x_{2}\right)$. Recall from Section 2 that this implies $D_{1}^{G}=1$. Now, $y$ will strictly prefer to buy from firm 2 when the firms locate at $\left(0, x_{2}^{\prime}\right)$, hence $D_{1}^{G^{\prime}}<1$. Since there exist a pair of strategies for which firms have different demands in the two games, the games cannot be equivalent.

The proof when $F$ is concave is an analog. Notice that arguments above can now be replicated for price strategies such that $y=x_{2}$ is an indifferent consumer.

Theorem 2. The games $G\left(C ; x_{1}, x_{2}\right)$ and $G^{\prime}\left(T ; x_{1}, x_{2}\right)$ are fully equivalent, where $C(z)$ is an arbitrary convex transport cost function, and $T(z)=C(1 / 2)-C(1 / 2-$ $z)$.

Proof. The proof of the theorem relies on the following two auxiliary results.

(a) The games $G\left(C ; x_{1}, x_{2}\right)$ and $G^{\prime \prime}\left(T ; x_{1}^{\prime}=x_{2}+1 / 2, x_{2}^{\prime}=1 / 2\right)$ are equivalent.

(b) The games $G^{\prime \prime}\left(T ; x_{1}^{\prime}=x_{2}+1 / 2, x_{2}^{\prime}=1 / 2\right)$ and $G^{\prime}\left(T ; x_{1}, x_{2}\right)$ are equivalent. ${ }^{3}$

We first prove (a). For an arbitrary consumer located at $x$, let $z_{i}$ and $z_{i}^{\prime}$ denote the distances between the consumer and firm $i$ in the games $G$ and $G^{\prime \prime}$, respectively. Since $x_{2}$ and $x_{1}^{\prime}$ are diametrically opposite each other on the circle (recall that $x_{1}^{\prime}=x_{2}+1 / 2$ ), then for any point $x$ on the circle, the distance from $x$ to $x_{1}^{\prime}$ plus the distance from $x$ to $x_{2}$ equals the distance from $x_{1}^{\prime}$, to $x_{2}$, that is, $1 / 2$. So $z_{2}=1 / 2-z_{1}^{\prime}$. A similar argument shows that $z_{1}=1 / 2-z_{2}^{\prime}$.

\footnotetext{
${ }^{3}$ Notice that $G^{\prime \prime}\left(T ; x_{1}^{\prime}=x_{2}+1 / 2, x_{2}^{\prime}=1 / 2\right)$ and $G^{\prime}\left(T ; x_{1}, x_{2}\right)$ coincide when locations are symmetric, i e, if $x_{1}=0$ and $x_{2}=1 / 2$
} 
From the above relations and the definition of $T(z)$, it is easily deduced that $T\left(z_{1}^{\prime}\right)=C(1 / 2)-C\left(z_{2}\right)$ and $T\left(z_{2}^{\prime}\right)=C(1 / 2)-C\left(z_{1}\right)$. Consequently, the sign of $\left[p_{1}+C\left(z_{1}\right)\right]-\left[p_{2}+C\left(z_{2}\right)\right]$ and the sign of $\left[p_{1}+T\left(z_{1}^{\prime}\right)\right]-\left[p_{2}+T\left(z_{2}^{\prime}\right)\right]$ are the same (in fact both expressions are equal). This implies that any consumer who prefers firm 1 in the game $G$ also prefers firm 1 in the game $G^{\prime \prime}$. Therefore each firm faces the same demand in the two games, and the two games are equivalent.

Now (b) follows immediately from Lemma 1, one only has to notice that in either game the distance between firms is equal to $x_{2}$.

Since $G$ is equivalent to $G^{\prime \prime}$ and $G^{\prime \prime}$ is equivalent to $G^{\prime}$ it follows that $G$ and $G^{\prime}$ are equivalent.

Corollary 3. The location-then-price game induced by $C$ is fully equivalent to the game induced by $T^{4}$

The equivalence result hinges on two key points. On one hand, it is necessary to find the appropriate transformation of the cost function which must take into account the maximum distance that a consumer may travel. On the other hand, the market is assumed circular which allows one to find, for any $x$ facing $C$, a consumer $\bar{x}$ facing $T$ such that $u\left(x, x_{1}\right)-u\left(x, x_{2}\right)=u\left(\bar{x}, x_{1}\right)-u\left(\bar{x}, x_{2}\right)$. Hence, it is important to assess the impact of changing the way the market is modeled.

In the linear market the maximum distance that a consumer may travel is 1 , and consequently, the appropriate transformation is $T(z)=C(1)-C(1-z)$. For this transformation the next example illustrates the failure of the equivalence result. Consider the strategy profile $x_{1}=0.25, x_{2}=0.75, p_{1}=2$ and $p_{2}=3$. For $C(z)=$ $10 z^{2}$ we have $D_{1}=0.6$ whereas for $T(z)=10\left(2 z-z^{2}\right)$ we have $D_{1}=0.533$.

\section{Oligopolistic competition}

In this section we suppose that $n$ firms, equi-spaced around the circle, compete in prices. Without loss of generality, we assume that firm $i$ is located at $x_{i}=(i-1) / n$. Notice that in this model there is no competition in locations. ${ }^{5}$ This assumption is appropriate when location is regulated.

\footnotetext{
${ }^{4}$ In De Frutos et al (1999) this argument is used to show that the game induced by the concave transport cost function $T(z)=z-z^{2}$ has a unique subgame perfect equilibrium The equilibrium strategies are $x_{1}=0, x_{2}=1 / 2, p_{1}=p_{2}=1 / 4$ Notice that these strategies constitute the unique subgame perfect equilibrium of the game induced by $C(z)=z^{2}$

${ }^{5}$ Economides (1989) has shown that equidistant locations is a subgame perfect equilibrium of the game induced by the cost function $C(z)=z^{2}$ irrespective of the number of firms in the market For an excellent review of oligopolistic competition in the circle model see Chapter 6 in Anderson et al (1992)
} 
With oligopolistic competition the demand of firm $i$ is the intersection of the $(n-1)$ market demands resulting from duopolistic competition between firm $i$ and each of the $(n-1)$ other firms operating in the market, i.e., $D_{i}=\cap D_{i j}, j \neq i$. If transport costs are convex, recall Section 2, then $1 / 2+x_{i} \notin D_{i j}$ for any $j$. If they are concave, then $x_{j} \notin D_{i j}$ for any $j$. In the former case, $D_{i}$ is a connected set whereas in the latter case it may not be connected. These statements rely on the following mathematical fact: if the intersection of arcs of the circle is non connected then the union of these arcs is the entire circle. With convex transport costs, the union of the duopolistic demands cannot be the entire circle. In contrast, when transport costs are concave the union of the duopolistic demands may be the entire circle, since, a priori, it is not possible to single out a point on the circle that does not belong to any of those demands. We now provide an example where this is indeed the case.

Let four firms compete in the market charging prices $p_{1}=0.1, p_{2}=p_{4}=0.23$, and $p_{3}=0.25$. Transport costs are given by $T(z)=C(1 / 2)-C(1 / 2-z)$, where $C(z)=z^{2}$. Straightforward computations show that $D_{1}$ is the non-connected set [0, $0.21) \cup(0.36,0.39) \cup(0.6,0.63) \cup(0.78,1)$. Notice that firm 1 shares one indifferent consumer with each of its competitors, we hence have non-localized competition as firm 1 is in direct competition with more than two other firms. In contrast, when the transport cost function is $C(z)=z^{2}$, we have localized competition as $D_{1}=(0.61,1) \cup[0,0.38)^{6}{ }^{6}$

The above example shows that the equivalence result does not hold under oligopolistic competition unless further restrictions are imposed. Nevertheless, it suggests that when competition is indeed localized some sort of equivalence result may hold. This turns out to be the case. We now provide a sufficient condition to guarantee localized competition which will allow us to obtain an equivalence result.

Assumption L. No consumer is willing to travel more than $1 / n^{7}$.

Assumption (L) holds when there exists limited consumer information. ${ }^{8}$ It implies

\footnotetext{
${ }^{6}$ The fact that competition is localized when transport costs are convex but may not be localized when they are concave is pointed out in Anderson et al (1992)

${ }^{7}$ The maximum distance that a consumer may now travel is $1 / n$ Consequently, the natural transformation to get equivalent price games is $T(z)=C(1 / n)-C(1 / n-z)$ Unfortunately, this transformation depends on the number of firms, and this could make it more difficult to do comparative statics in the number of firms for a given concave transport cost

${ }^{8}$ Competition in a circular market when consumers have limited information has been studied in Peitz (1999) He provides the following explanation 'Consider a market of differentiated goods which are ordered as 1, 2, , $n$ Each pair of goods $(i, i+1)$ is observed by an equal share of consumers $1 / n$, i e consumers draw a good $i$ with probability $1 / n$ (independently over consumers) and then with probability $1 / 2$ one of the neighboring goods This consumer information can be best understood as arising when consumers who enter a shop select only among a pair of neighboring goods they encounter in the shelf'
} 
that consumers make comparisons only between the two firms that are closest to them and then purchase from one of them.

Proposition 4. If Assumption (L) holds, for any convex transport cost $C(z)$ there exists a concave transport cost, $T(z)=C(1 / n)-C(1 / n-z)$, such that the price games induced by these two transport cost functions are fully equivalent.

Proof. To prove the proposition it is enough to show that the demand of firm $i$ under $C$ coincides with the demand of firm $i$ under $T$, i.e. $D_{i}^{C}(p)=D_{i}^{T}(p)$. Moreover, since competition is assumed localized, demand of firm $i$ will only depend on the prices charged by firm $i$ and by its two neighboring firms, i.e., firms $i-1$ and $i+1$.

Let $x$ be a consumer located between firm $i$ and one of its two neighboring firms. Let $z_{i}$ (respectively, $z_{j}$ ) represent the distance between $x$ and firm $i$ (respectively, firm $j, j=i-1, i+1$ ). Notice that $z_{i}+z_{j}=1 / n$. It is now straightforward to see that

$$
p_{i}+C\left(z_{i}\right)-\left(p_{j}+C\left(z_{j}\right)\right)=p_{i}+T\left(z_{i}\right)-\left(p_{j}+T\left(z_{j}\right)\right) .
$$

Therefore, any consumer who prefers to buy from firm $i$ when transport cost are convex will also prefer to buy from this firm when transport cost are concave. Consequently, $D_{i}^{C}(p)=D_{i}^{T}(p)$.

Corollary 5. Under equidistant locations, if assumption $(L)$ holds, then the transport cost functions $C(z)=z^{2}$ and $T(z)=\frac{2}{n} z-z^{2}$ induce two fully equivalent price games. Moreover, these two games have a unique symmetric equilibrium given by $p^{*}=(1 / n)^{2}$.

Proof. The result follows from previous proposition and from Corollary 1 in Economides (1989).

\section{Acknowledgements}

The authors have benefitted from comments and suggestions by Simon P. Anderson, Xavier Martínez, Inés Macho, David Pérez-Castrillo, and Roberto Serrano. Valuable suggestions by the editor and an anonymous referee have substantially improved the paper. M.A. de Frutos acknowledges financial support from the Spanish Ministry of Education grant BEC2000-0173 and X. Jarque the financial support from the DIGICYT under projects PB96-1153 and SEC20000684, and from the Government of Catalonia under project SGR99-00349. 


\section{References}

Anderson, S.P., 1986. Equilibrium existence in the circle model of product differentiation. London Papers in Regional Science Series 16, 19-29.

Anderson, S.P., de Palma, A., Thisse, J.F., 1992. Discrete Choice Theory of Product Differentiation. MIT Press, Cambridge, Massachusetts.

De Frutos, M.A., Hamoudi, H., Jarque, X., 1999. Equilibrium existence in the circle model with linear quadratic transport cost. Regional Science and Urban Economics 29, 605-615.

Economides, N., 1989. Symmetric equilibrium existence and optimality in a differentiated product market. Journal of Economic Theory 47, 178-194.

Myerson, R., 1991. Game Theory Analysis of Conflict. Harvard University Press, Cambridge, Massachusetts.

Peitz, M., 1999. The circular road revisited: uniqueness and supermodularity. Research in Economics 53 (4), 405-420.

Salop, S.C., 1979. Monopolistic competition with outside goods. Bell Journal of Economics 10, 141-156. 